ORIGINAL ARTICLE

\title{
Molecular Mechanisms of Quercitrin-induced Apoptosis in Non-small Cell Lung Cancer
}

\author{
Zeynep Birsu Cincin, ${ }^{\mathrm{a}}$ Miray Unlu, ${ }^{\mathrm{b}}$ Bayram Kiran, ${ }^{\mathrm{c}}$ Elif Sinem Bireller, ${ }^{\mathrm{a}}$ Yusuf Baran, ${ }^{\mathrm{b}}$ and \\ Bedia Cakmakoglu \\ ${ }^{\mathrm{a}}$ Department of Molecular Medicine, Istanbul University, Institute of Experimental Medical Research, Capa, Istanbul, ${ }^{\mathrm{b}}$ Department of Molecular Biology and \\ Genetics, Izmir Institute of Technology, Urla, Izmir, ${ }^{\mathrm{c} D e p a r t m e n t ~ o f ~ B i o l o g y, ~ K a s t a m o n u ~ U n i v e r s i t y, ~ К а s t a т о п и, ~ T u r k e y ~}$
}

Received for publication February 17, 2014; accepted August 12, 2014 (ARCMED-D-14-00106).

Background and Aims. Quercitrin (QR; quercetin-3-O-rhamnoside) has been used previously as an antibacterial agent and has been shown to inhibit the oxidation of low-density lipoproteins and prevent an allergic reaction. Furthermore, it was demonstrated that quercitrin exerts protective effects against $\mathrm{H}_{2} \mathrm{O}_{2}$-induced dysfunction in lung fibroblast cells. However, the mechanisms of quercitrin effects on cancer cell proliferation and apoptosis is not well understood. The aim of this study is to investigate the cytotoxic and apoptotic effects of quercitrin and the molecular mechanisms of quercitrin-induced apoptosis in non-small cell lung cancer (NSCLC) cell lines.

Methods. Time- and dose-dependent antiproliferative and apoptotic effects of quercitrin determined by WST-1 cell proliferation assay, lactate dehydrogenase (LDH) cytotoxicity assay, determination of nucleosome enrichment factor, changes in caspase-3 activity, loss of mitochondrial membrane potential (MMP) and also the localization of phosphatidylserine in the plasma membrane. Changes in whole genome gene expression levels were examined by Illumina Human HT-12v4 beadchip microarrays.

Results. There were significant increases in caspase- 3 activity, loss of MMP, and increases in apoptotic cell population in response to quercitrin in A549 and NCI-H358 NSCLC cells in a time- and dose-dependent manner.

Conclusion. Our results demonstrated that genes involved in leukocyte transendothelial migration, cell adhesion and phosphatidylinositol signaling system pathways were the most statistically significant pathways in NCI-H358 and A549 cells. These results revealed that quercitrin has antiproliferative and apoptotic effects on lung cancer cells by modulating the immune response. After confirming its anticarcinogenic effects in vivo, quercitrin could be a novel and strong anticancer agent against NSCLC. (C) 2014 IMSS. Published by Elsevier Inc.

Key Words: Non-small cell lung cancer, Quercitrin, Apoptosis, Microarray.

\section{Introduction}

Lung cancer is the leading cause of cancer-related deaths worldwide among women and men, which is estimated at $>1,000,000$ deaths each year (1). Lung cancer is clinically divided into two classes, non-small cell lung cancer

Address reprint requests to: Dr., Prof., Bedia Cakmakoglu, Istanbul University, Institute of Experimental Medical Research, Department of Molecular Medicine, Capa, Istanbul, Turkey; Phone: +90 212 414200033305; FAX: +90 212 5324171; E-mail: bedia@istanbul.edu.tr
(NSCLC) and small cell lung cancer (SCLC) due to their tendency to metastasize and response to existing therapies (2). NSCLC accounts for $80 \%$ of all lung cancer cases, with adenocarcinoma being the major subtype (3). NSCLC is less responsive to chemotherapy and as such has been the main focus of current research on investigating new drugs for its therapy (4). The prognosis of lung cancer is poor because of the late diagnosis at advanced stage (5). Although $70 \%$ of newly diagnosed patients present with locally advanced or metastatic lung cancer and require systemic treatment, chemotherapeutic drugs and molecular 
targeted therapies have limitations because of dose-limiting toxicities and acquisition of drug resistance (6-8). Recent studies have focused on dietary agents to develop effective prevention strategies for lung cancer including nutraceuticals, which are non-toxic, cost-effective, and physiologically bioavailable $(9-11)$.

Flavonoids are widely found in fruits and vegetables and exert various biological activities that are mainly related to their abilities to inhibit enzymes, their antioxidant properties, and to their effects on immune responses (12). These properties could explain the beneficial effects that flavonoid intake exerts in different human pathologies including hypertension, inflammatory conditions, and cancer (13). Quercitrin is the most abundant bioflavonoid compound, which is mainly present in the glycoside form (14). The sugar portion is bound to the aglycone portion in quercitrin that increases its solubility and improves absorption. Recent studies demonstrated that quercitrin exhibits a scavenger and anti-oxidant role; consequently, quercitrin has become the center of attention for its potential anti-carcinogenic activity (15). Although different studies have indicated that quercitrin is a potent antioxidant, the action of this compound is not well understood (16-18). Hence, we proposed to investigate whether quercitrin has apoptotic and antiproliferative effects in NSCLC and lung fibroblast cells and examined its effects on genome-wide expression profiling.

\section{Materials and Methods}

\section{Cell Culture}

The bronchioalveolar carcinoma cell line NCI-H358 (ATCC number: CRL-5807) and lung fibroblast cell line MRC-5 (ATCC number: CCL-171) as a control line were obtained from the ATCC (American Type Culture Collection, Manassas, VA). The A549 adenocarcinoma cell line was kindly provided by Dr. Yusuf Baran (Department of Molecular Biology and Genetics, IYTE). NCI-H358 cell line was cultured in an RPMI-1640 medium (ATCC modification) containing $1 \%$ penicillin/streptomycin and $10 \%$ fetal bovine serum at $37^{\circ} \mathrm{C}$ in $5 \% \mathrm{CO}_{2}$. The A549 and MRC- 5 cell lines were cultured in MEM- $\alpha$ medium containing $1 \%$ penicillin/streptomycin and $10 \%$ fetal bovine serum at $37^{\circ} \mathrm{C}$ in $5 \% \mathrm{CO}_{2}$.

\section{Measurement of Cell Growth and Cytotoxicity}

To detect effects on cell viability after quercitrin treatment, WST-1 cell proliferation assay performed. The WST-1 conversion assay (Roche, Germany) is based on the mitochondrial function of intact cells, which enables them to metabolize the stable tetrazolium salt WST-1 (4-[3-(4-iodophenyl)-2-(4-nitrophenyl)-2H-5-tetrazolio]1,3-benzene disulfonate) to a soluble violet formazan product. Ninety-six-well plates containing $100 \mu \mathrm{l}$ of the growth medium were seeded at $1 \times 10^{4}$ cells/well in the absence or presence of increasing concentrations of quercitrin and then incubated at $37^{\circ} \mathrm{C}$ in $5 \% \mathrm{CO}_{2}$ for 24,48 and $72 \mathrm{~h}$. After the incubation period, cells were treated with $10 \mu \mathrm{l}$ of WST-1 for $4 \mathrm{~h}$. Color development was measured at $450 \mathrm{~nm}$ using a Multiscan ELISA reader (Thermo Fisher Scientific, Germany). Viability was calculated by subtraction of the mean values without WST-1 from those with WST-1 substrate and expressed as a percentage of control. Data were confirmed by an additional three independent experiments.

Dose- and time-dependent cytotoxic effects of quercitrin were colorimetrically determined with a "CytoTox 96R Non-Radioactive Cytotoxicity Assay" kit from Promega (Madison, WI). Cells were treated as described for the WST-1 assay. Culture medium $(10 \mu \mathrm{l})$ was then transferred to a 96-well microtiter plate. The levels of lactate dehydrogenase (LDH) were determined by adding $50 \mu$ freshly prepared Substrate Mix, incubating in darkness at room temperature for $30 \mathrm{~min}$, adding $50 \mu \mathrm{l}$ STOP Solution, and measuring optical density (OD) at $490 \mathrm{~nm}$ with a microplate reader (Bio-Rad, Hercules, CA). Data were confirmed by three further independent experiments.

\section{Detection of Apoptotic Nucleosomes and Necrotic DNA Release}

The Cell Death Detection ELISAPLUS (CDDE) kit is a photometric enzyme-immunoassay for the qualitative and quantitative in vitro determination of DNA in the supernatant of treated cells that indicates necrosis and cytoplasmic histone-associated DNA fragments (mono- and oligonucleosomes) indicating apoptosis (Roche, Germany). Application according to manufacturer's instructions provides concurrent detection of apoptosis and necrosis in the same well. Color development of samples was considered as an enrichment factor of the amount of DNA fragments in the cytoplasm or cell supernatant, indicating apoptosis or necrosis, respectively, and expressed relative to untreated cells. In order to investigate possible interferences with the assay, particles were added to the cells in a concentration two fold higher than the final concentration used in the above experiment $\left(160 \mu \mathrm{g} / \mathrm{cm}^{2}\right)$. Subsequently, the samples were centrifuged (10 min, $200 \mathrm{~g})$ in conformance with the CDDE kit protocol. The supernatant was then mixed 1:1 with the lysate of $50 \mu \mathrm{m}$ quercitrin treated cells for 48 and $72 \mathrm{~h}$ using a lysis buffer, which is included in the kit, and analysed according to the manufacturer's instructions.

\section{Analysis of Phosphatidylserine Exposure and Cell Permeability}

During apoptotic cell death, phosphatidylserine (PS), a phospholipid component of the inner-leaflet of cell membranes, becomes available at the cell surface. This early marker of apoptotic cell death can be detected by staining with the green fluorescent dye Annexin V-FITC (BD 
Pharmingen, Germany), a fluorochrome-conjugated $\mathrm{Ca}^{2+}$ dependent PS-binding protein. In combination with the 7AAD-DNA-staining, dye exclusion of vital cells permits discrimination between apoptotic and necrotic cells. Cells treated in 24-well dishes were centrifuged (200 g, $5 \mathrm{~min}$, $4^{\circ} \mathrm{C}$ ), washed with HBSS-/- (GIBCO, Germany) and stained with $150 \mu \mathrm{l}$ of buffer $(10 \mathrm{mmol} \mathrm{HEPES} / \mathrm{NaOH}$ pH 7.4, $150 \mathrm{mmol} \mathrm{NaCl}, 5 \mathrm{mmol} \mathrm{KCl,} 2 \mathrm{mmol} \mathrm{CaCl}$ ) containing $5 \mu \mathrm{l}$ Annexin V-FITC $(1 \mathrm{mg} / \mathrm{mL})$ and $1.5 \mu \mathrm{mol}$ 7 -AAD at $37^{\circ} \mathrm{C}$. After $10 \mathrm{~min}$, an additional $500 \mu \mathrm{l}$ of ice-cold buffer was added and cells were harvested by scraping the ice. After centrifugation $\left(200 \mathrm{~g}, 5 \mathrm{~min}, 4^{\circ} \mathrm{C}\right)$, cells were suspended in $250 \mu \mathrm{l}$ buffer and immediately analysed by flow cytometry using the green-collecting fluorescent channels FL-1 for Annexin V-FITC and FL-3 for 7-AAD, as described above. Quadrant separation in the fluorescent channels FL-1 (Annexin V-FITC) vs. FL-3 (7-AAD) represents events of necrotic and late apoptotic cells, respectively, which are 7-AAD positive $(\mathrm{Q} 1+\mathrm{Q} 2)$; apoptotic cells (Q3, Annexin V-FITC positive and 7-AAD negative) and viable cells ( $\mathrm{Q} 4$, fluorescent negative) expressed as percentage of total cells.

\section{Analysis of Caspase-3 Enzyme Activity}

Changes in caspase- 3 enzyme activity of the cells are an important sign of apoptosis. These were examined by caspase-3 colorimetric assay kit (BioVision Research Products, Milpitas, CA). This assay is based on spectrophotometric detection of the chromophore p-nitroanilide (pNA) after cleavage from the labeled substrate DEVDpNA, which can be recognized by caspase-3. In short, the cells $\left(5 \times 10^{5}\right.$ cells $/ 2 \mathrm{~mL} /$ well $)$ induced apoptosis and were collected by centrifugation at $1000 \mathrm{rpm}$ for $10 \mathrm{~min}$. Cells were lysed by adding $50 \mu \mathrm{l}$ of chilled cell lysis buffer and incubated on ice for $10 \mathrm{~min}$ before centrifugation at $10000 \mathrm{~g}$ for $1 \mathrm{~min}$. Supernatants were transferred to new Eppendorf tubes, and the reaction mixture was prepared in 96-well plates by adding $50 \mu$ of reaction buffer (containing $10 \mathrm{mmol}$ DTT), $50 \mu \mathrm{l}$ of sample, and $5 \mu \mathrm{l}$ of DEVD-pNA substrate and incubated for $2 \mathrm{~h}$ at $37^{\circ} \mathrm{C}$ in $\mathrm{CO}_{2}$ incubator. After incubation, the plate was read under $405 \mathrm{~nm}$ wavelengths using an ELISA reader (Thermo Electron Corporation Multiskan Spectrum, Finland). The absorbance values were normalized to protein concentrations determined by a Bradford assay, as described previously.

\section{Determination of Loss in Mitochondrial Membrane Potential}

We examined the loss of MMP, another important sign of apoptosis, in response to quercitrin treatment for $48 \mathrm{~h}$ in A549 and MRC5 cells by JC-1 Mitochondrial Membrane Potential Detection Kit (Cayman Chemicals, Ann Arbor, MI). This kit uses JC-1, a unique cationic dye, to signal the loss of the MMP. JC-1 accumulates in the mitochondria, which stain red in nonapoptotic cells, whereas in apoptotic cells, the MMP collapses; thus, the JC-1 remains in the cytoplasm as a monomer that stains green under fluorescent light. In brief, the cells $\left(5 \times 10^{5}\right.$ cells $\left./ 2 \mathrm{~mL}\right)$ induced apoptosis and were collected by centrifugation at $1000 \mathrm{rpm}$ for $10 \mathrm{~min}$. Supernatants were removed, pellets were homogenized using $200 \mu \mathrm{l}$ of medium, and $20 \mu \mathrm{l}$ of JC-1 dye was added onto the cells. Cells were then incubated at $37^{\circ} \mathrm{C}$ in $5 \% \mathrm{CO}_{2}$ for $30 \mathrm{~min}$ and centrifuged at $400 \mathrm{~g}$ for $5 \mathrm{~min}$. Supernatants were removed, and $200 \mu \mathrm{l}$ of assay buffer was added onto the pellets and vortexed. This step was repeated once more. Afterwards, all pellets were resuspended with $320 \mu \mathrm{l}$ assay buffer and $100 \mu \mathrm{l}$ from each was added into the 96-well plate as triplicates. In healthy nonapoptotic cells, the aggregate red form has absorption/emission maxima of 560/595 nm, whereas in apoptotic cells the monomeric green form has absorption/ emission maxima of $485 / 535 \mathrm{~nm}$. The plate was read at these wavelengths using a fluorescence Elisa reader (Thermo Varioskan Spectrum, Finland). The resulting green/red (485/560) values were calculated to determine the changes in MMP.

\section{RNA Extraction}

Total RNA was isolated from A549, NCI-H358, and MRC5 , and treated with $50 \mu \mathrm{mol}$ quercitrin for $48 \mathrm{~h}$ using High Pure RNA Isolation Kit (Roche) in accordance with the manufacturer's instructions. RNA concentrations were assessed with the NanoDrop (Thermo Scientific) spectrophotometer (260/280 $\mathrm{nm}$ ratios) and only the samples with an A260/A280 ratio between 1.9 and 2.1 were considered suitable for use.

\section{Microarray Hybridization}

Illumina Human HT-12v4 beadchip microarrays (containing 47,000 probes derived from the National Center for Biotechnology Information Reference Sequence [NCBI] RefSeq Release 38 [November 7, 2009] and other sources) (Illumina, Inc., San Diego, CA) were used to assess global gene expression for each sample. Five hundred nanograms of total RNA was amplified, converted to cRNA, fragmented, and then biotin-labeled using the Illumina TotalPrep RNA-amplification kit (Ambion, Life Technologies, Grand Island, NY). Then, $1.5 \mu \mathrm{g}$ of labeled cRNA was hybridized to each array in accordance with the Illumina whole-genome gene expression direct hybridization assay protocol, after which arrays were scanned using the Illumina BeadArray Reader. Images were processed and converted into signal intensities using the Illumina GenomeStudio software (Illumina, Inc.). The same software was used to perform hybridization quality control (QC). 

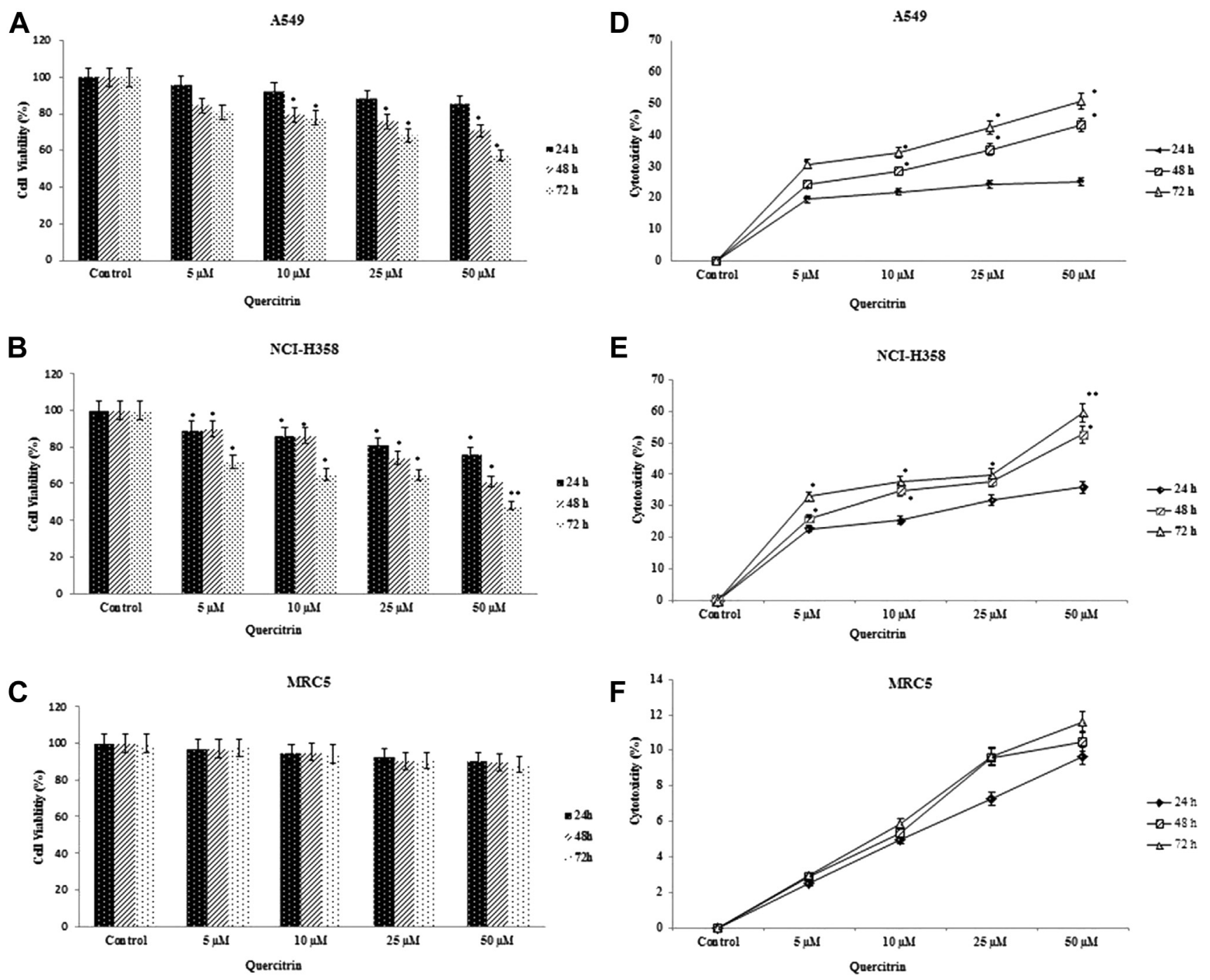

Figure 1. Effects of quercitrin on cell proliferation and cytotoxicity of A549 (A-D), NCI-H358 (B-E), and MRC-5 (C-F) cells. WST-1 and LDH assays were performed using triplicate samples in three independent experiments. Statistical significance was determined using two-way analysis of variance, $* p<0.05,{ }^{* *} p<0.01$ was considered significant. IC50 value of quercitrin was calculated from cell proliferation plots.

\section{Statistical Analysis}

The signal intensities corresponding to gene expression levels of individual arrays were background corrected and imported into text files using the Illumina Genome Studio. Hierarchical cluster analysis performed on datasets to evaluate the "proximity" between the time points. For data visualization, hierarchical clusters were constructed with the statistically significant $(p<0.05)$ genes. Computing a $p$ value for each gene assessed the statistical significance of the differential expression of genes. Genes were considered differentially expressed when logarithmic gene expression ratios in three independent hybridizations were $>1.5$ or $<0.66$, i.e., 1.5 -fold difference in expression level, and when the $p$ values were $<0.05$. Using the Kyoto Encyclopedia of Genes and Genomes (KEGG) and Ingenuity Pathway Analysis (IPA; Ingenuity
Systems, Redwood City, CA), gene ontology and pathway analyses were performed to consider the biological meaning of differential expression of genes between the data points.

\section{Results}

Quercitrin Inhibited Proliferation of A549 and NCI-H358 NSCLC Cell Lines in a Time- and Dose-dependent Manner

To determine the antiproliferative effects of quercitrin on A549 and NCI-H358 NSCLC, and MRC-5 normal lung fibroblast cells as a control line, the cells were incubated with increasing concentrations of quercitrin for 24, 48, $72 \mathrm{~h}$ using WST-1 cell proliferation assay. The results of these assays showed that there were time- and dose- 

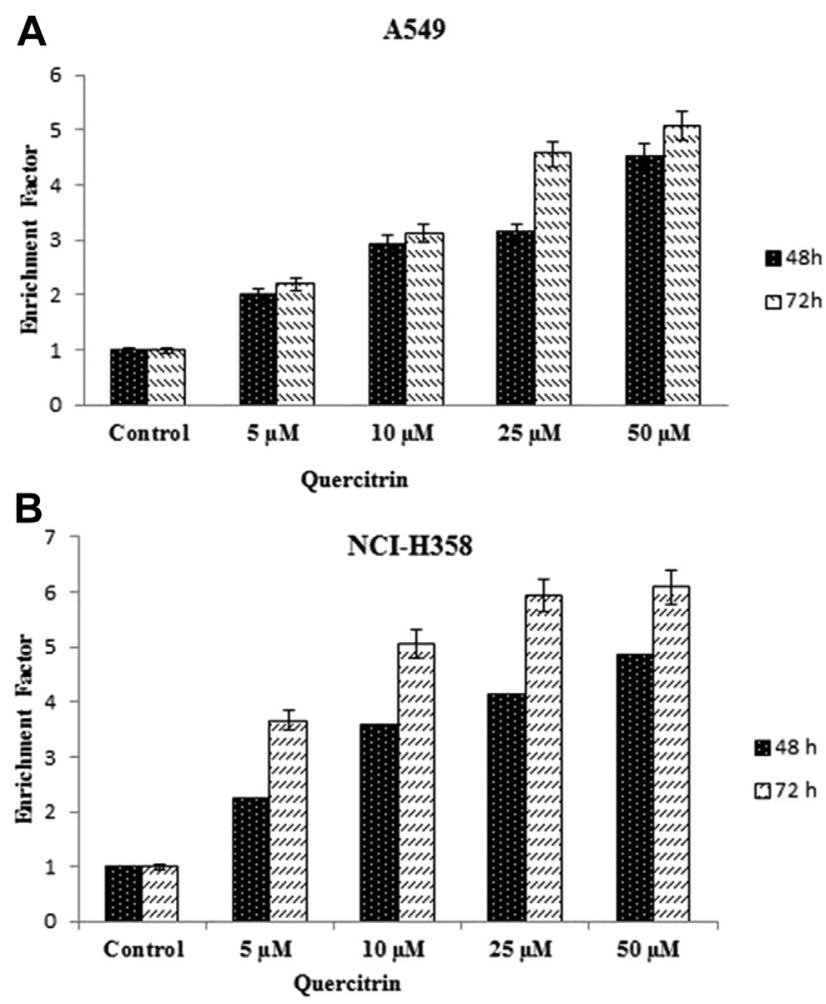

Figure 2. Effects of quercitrin on apoptosis-related nucleosomal enrichment factor of A549 (2A), NCI-H358 (2B), cells. The Cell Death detection plus ELISA were performed using triplicate samples in at least two independent experiments. Statistical significance was determined using twoway analysis of variance, and $p<0.05$ was considered significant.

dependent decreases in cell proliferation only with NSCLC lines compared with controls (Figures 1A-1C).

IC50 values of quercitrin for 24,48 and $72 \mathrm{~h}$ were calculated from both cell proliferation and cytotoxicity plots and found to be $50 \mu \mathrm{mol}$, respectively (Figure 1).

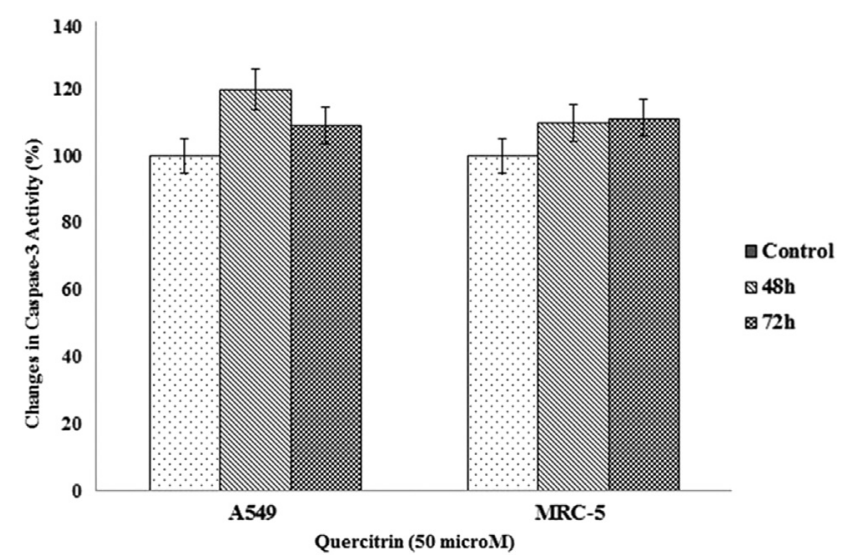

Figure 3. Effects of quercitrin on caspase 3 activity in A549 and MRC5 cells. Changes in caspase- 3 enzyme activity in response to increasing concentrations of quercitrin in A549 and MRC-5 cells. The results are the means of two independent experiments. $p<0.05$ was considered significant.

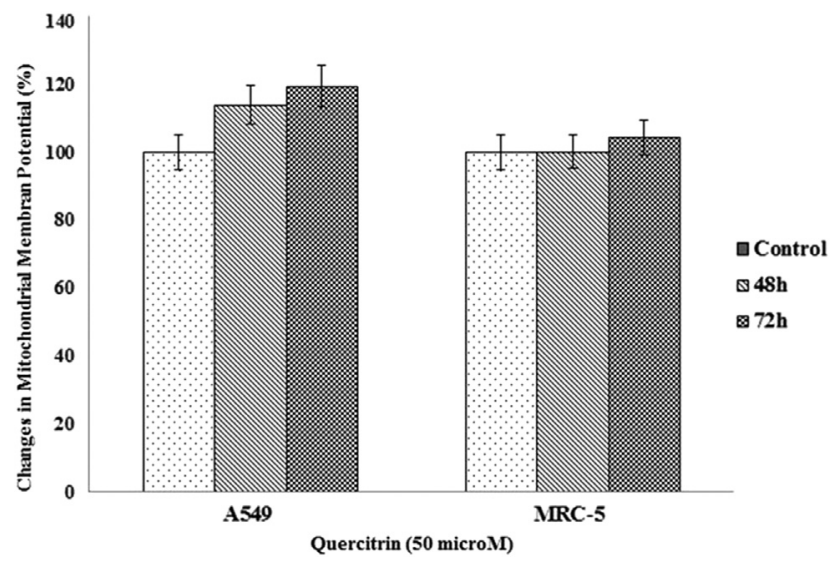

Figure 4. Effects of quercitrin on membrane potential in A549 and MRC5 cells. Loss of mitochondrial membrane potential in response to increasing concentrations of quercitrin in A549 and MRC-5 cells. The results are the means of two independent experiments. $p<0.05$ was considered significant.

Quercitrin Has Cytotoxic Effects on A549 and NCI-H358 NSCLC Cell Lines in a Time- and Dose-dependent Manner

To determine the cytotoxic effects of quercitrin on A549 and NCI-H358 NSCLC, and MRC-5 normal lung fibroblast cells as a control line, the cells were incubated with increasing concentrations of quercitrin for $24,48,72 \mathrm{~h}$ using an LDH assay. The results of these assays showed that there were time- and dose-dependent increases in cytotoxicity only in the NSCLC cell lines compared with controls (Figures 1D-1F). IC50 values of quercitrin for 24, 48 and $72 \mathrm{~h}$ were calculated from both cell proliferation and cytotoxicity plots and found to be $50 \mu \mathrm{mol}$, respectively (Figure 1).

\section{Quercitrin Increases Nucleosomal Enrichment Factor in a Time- and Dose-dependent Manner}

To determine the apoptotic effects of quercitrin on A549 and NCI-H358 cells, these cells were incubated with increasing concentrations of quercitrin for 48 and $72 \mathrm{~h}$, and changes in nucleosomal enrichment factor (EF) were analyzed. There were subsequently 4.53 - and 5.07-fold increases in EF in response to 48 and $72 \mathrm{~h}$ incubation with $50 \mu \mathrm{mol}$ quercitrin on A549 cells as compared with untreated cells (Figure 2A). Also, there were 4.87- and 6.08-fold increases in EF in response to 48 and $72 \mathrm{~h}$ incubation with $50 \mu \mathrm{mol}$ quercitrin on NCI-H358 cells when compared with untreated cells (Figure 2B).

\section{Quercitrin Increases Caspase-3 Enzyme Activity in a} Time- and Dose-dependent Manner

To determine the apoptotic effects of quercitrin on A549 and MRC-5 cells, these cells were incubated with 
A
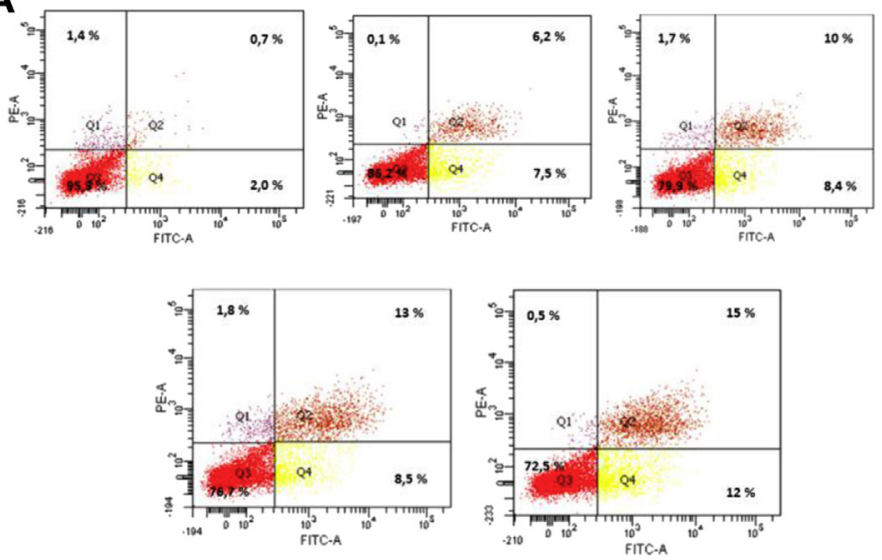

C
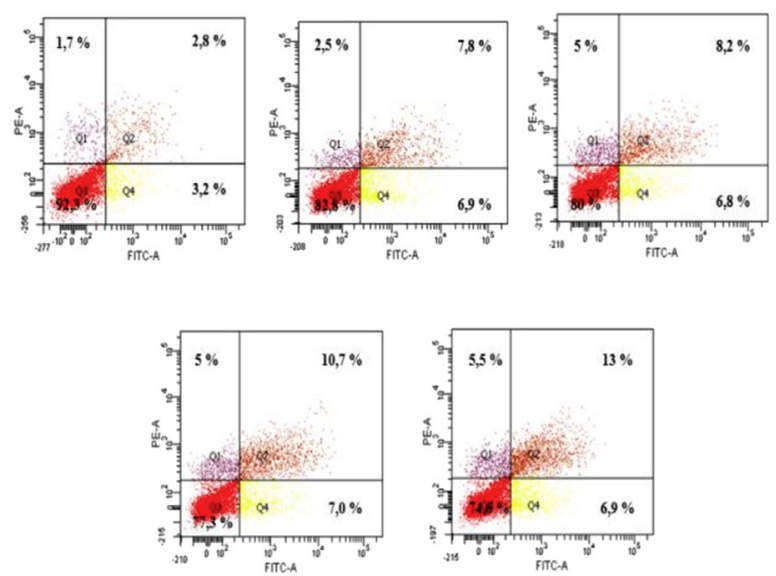

B

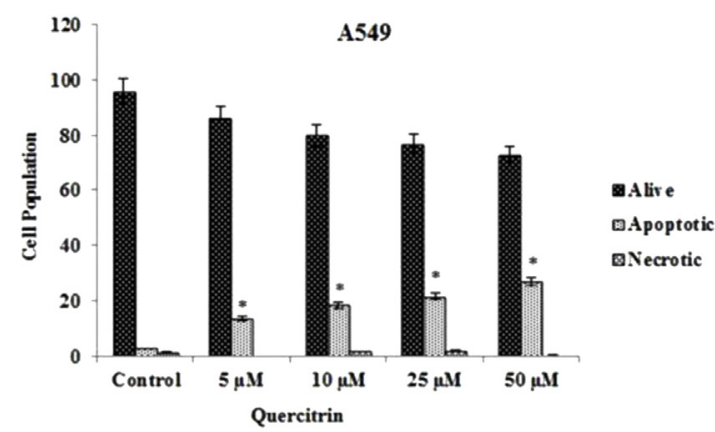

D

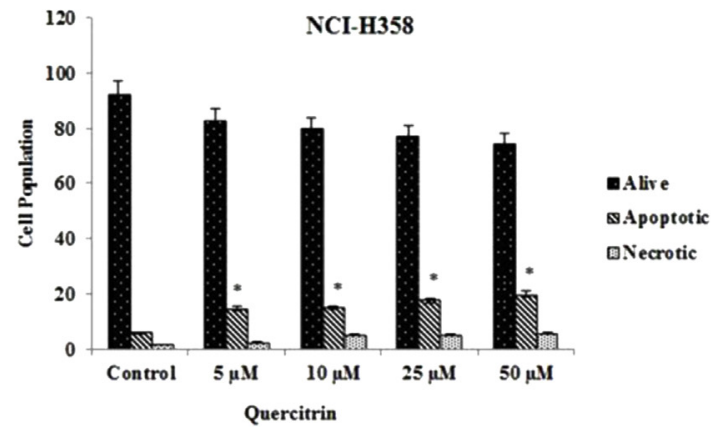

Figure 5. FITC AnnexinV/PI double staining of A549 (5A) cells NCI-H358 (5B) cells treated with increasing concentrations of quercitrin. Early apoptotic cells labelled with Annexin- $\mathrm{V}(+) / \mathrm{PI}(-)$ and apoptotic cells labelled with Annexin- $\mathrm{V}(+) / \mathrm{PI}(-)$ in flow cytometric graphics. The results are the means of two independent experiments. $p<0.05$ was considered significant. Cell population graphs shown in Figures 5B-5D.

increasing concentrations of quercitrin for $48 \mathrm{~h}$ and changes in caspase-3 enzyme activities were analyzed. There was a 1.19 -fold increase in caspase- 3 activity in response to $48 \mathrm{~h}$ incubation with $50 \mu \mathrm{mol}$ quercitrin on A549 cells when compared with untreated cells (Figure 3). There was no change in caspase 3 activity of MRC- 5 cells under the same conditions as with A549 cells (Figure 3). The fact that quercitrin induced apoptosis in a dose-dependent manner may be related with caspase- 3 activity.

\section{Quercitrin Induces the Loss of Mitochondrial Membrane Potential in a Time- and Dose-dependent Manner}

To assess the loss of MMP, A549 and MRC5 cells were exposed to $50 \mu \mathrm{mol}$ quercitrin for $48 \mathrm{~h}$ and a JC-1 MMP assay was performed. The results of this assay revealed that there was a 1.14-fold increase in the loss of MMP in

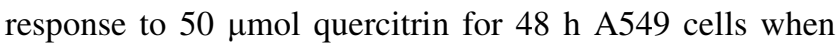
compared with untreated cells (Figure 4). There was no change for MMP potential of MRC-5 cells under the same conditions with A549 cells (Figure 4).
Quercitrin Causes Modulation of the Cell Membrane Resulting in the Translocation of PS from the Inner to the Outer Leaflet in a Time- and Dose-dependent Manner

To confirm the results of caspase- 3 activity and loss of MMP, FITC AnnexinV/PI double staining was performed in A549 (Figure 5A) and NCI-H358 (Figure 5B) NSCLC cell lines and MRC-5 normal lung fibroblast cells as a control line exposed to $50 \mu \mathrm{mol}$ quercitrin for $48 \mathrm{~h}$. The results demonstrated that $48 \mathrm{~h}$ incubation of A549 and NCI$\mathrm{H} 358$ cells with $50 \mu \mathrm{mol}$ quercitrin increased apoptotic cell death when compared with untreated control group (Figures 5C and 5D).

\section{Changes in Expression Levels of Whole Genome in Response to Quercitrin}

To determine the biologically relevant networks beyond canonical pathways, pathway analysis was performed for gene sets using IPA. The networks describe functional relationships between gene products based on 
Table 1. Top network

\begin{tabular}{|c|c|c|}
\hline Genes involved in network & Score & Network \\
\hline 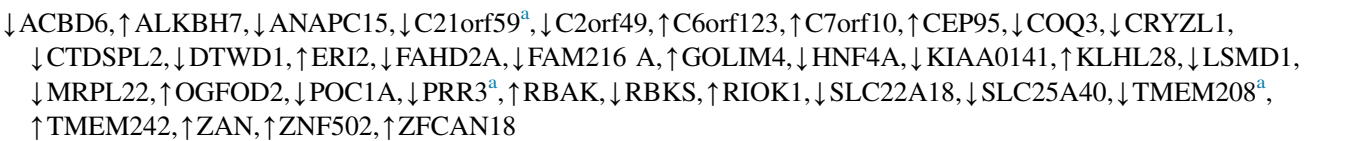 & 34 & $\begin{array}{l}\text { Cellular development, cell morphology, cellular } \\
\text { function and maintenance }\end{array}$ \\
\hline 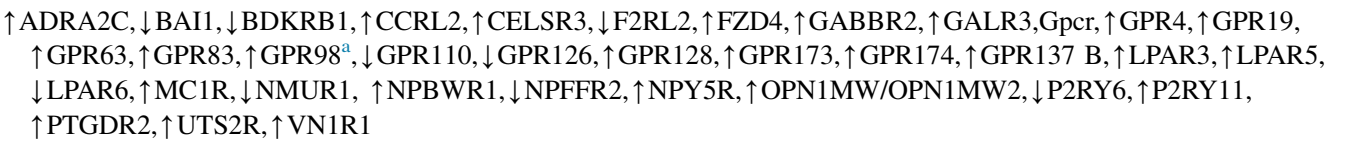 & 32 & $\begin{array}{l}\text { Cell-to-cell signaling and interaction, nervous system } \\
\text { development and function, carbohydrate metabolism }\end{array}$ \\
\hline 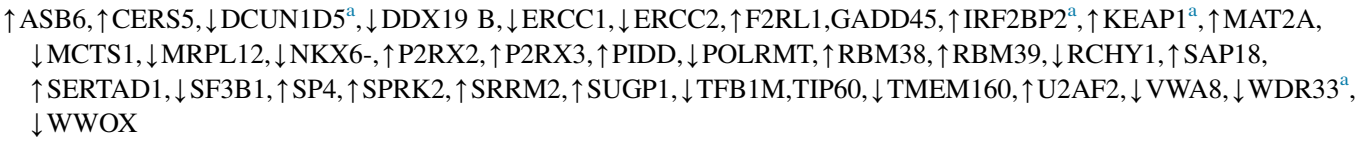 & 30 & $\begin{array}{l}\text { Developmental disorder, hereditary disorder, neurological } \\
\text { disease }\end{array}$ \\
\hline 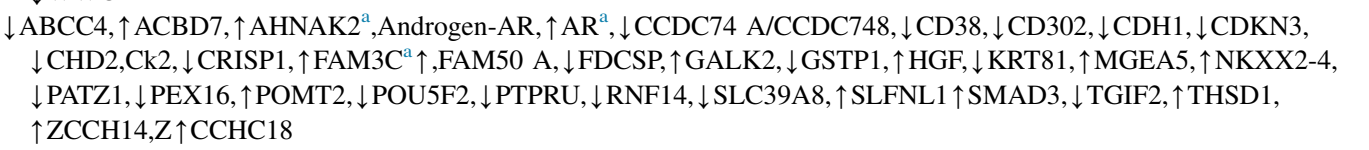 & 30 & Tumor morphology, cell morphology, cellular development \\
\hline 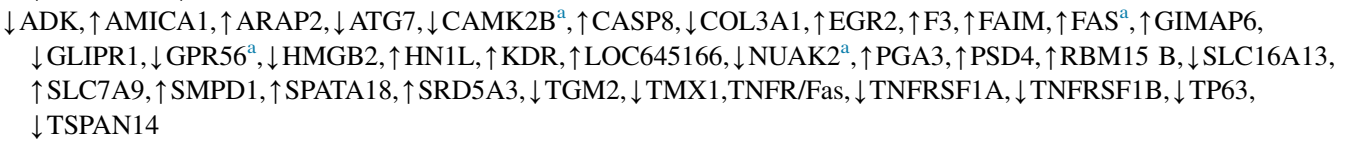 & 29 & $\begin{array}{l}\text { Lipid metabolism, small molecule biochemistry, cell death } \\
\text { and survival }\end{array}$ \\
\hline 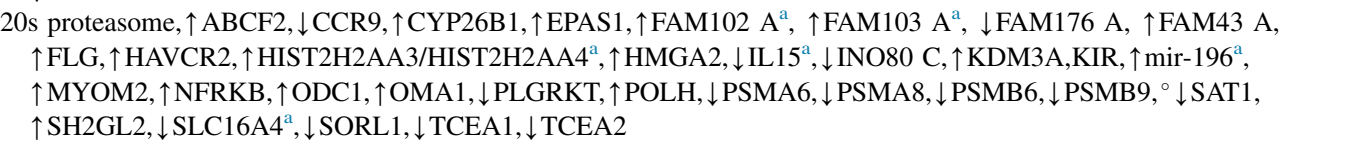 & 28 & $\begin{array}{l}\text { Molecular transport, small molecule biochemistry, cellular } \\
\text { development }\end{array}$ \\
\hline
\end{tabular}

${ }^{a}$ Literature-based clustering of differentially genes using an Ingenuity Pathway Analysis (IPA) after $48 \mathrm{~h}$ of $50 \mu$ mol quercitrin stimulation in A549 cells. According to IPA, three major pathways emerged: (I) cell signaling, molecular transport, (II) cellular assembly and organization and (III) cellular development. 
Table 2. Literature-based clustering of differentially genes using an Ingenuity Pathway Analysis (IPA) after $48 \mathrm{~h}$ of $50 \mu \mathrm{M}$ quercitrin stimulation in NCI-H358 cells ${ }^{\mathrm{a}}$

\begin{tabular}{lcc}
\hline Diseases and disorders & $\boldsymbol{p}$ & \# molecules \\
\hline Cancer & $1,30 \mathrm{E}-16-3,55 \mathrm{E}-03$ & 1104 \\
Gastrointestinal disease & $1,45 \mathrm{E}-12-1,43 \mathrm{E}-03$ & 487 \\
Reproductive system disease & $3,95 \mathrm{E}-08-3,11 \mathrm{E}-03$ & 378 \\
Respiratory disease & $1,15 \mathrm{E}-07-1,39 \mathrm{E}-03$ & 247 \\
Endocrine system disorders & $2,76 \mathrm{E}-06-3,11 \mathrm{E}-03$ & 184 \\
Molecular and cellular functions & & \\
$\quad$ Cell death and survival & $5,99 \mathrm{E}-08-3,57 \mathrm{E}-03$ & 875 \\
$\quad$ Cellular growth and proliferation & $1,87 \mathrm{E}-07-3,53 \mathrm{E}-03$ & 868 \\
$\quad$ Cell cycle & $1,31 \mathrm{E}-06-3,29 \mathrm{E}-03$ & 383 \\
Cellular development & $3,42 \mathrm{E}-06-3,53 \mathrm{E}-03$ & 779 \\
Cell morphology & $7,73 \mathrm{E}-06-3,20 \mathrm{E}-03$ & 389 \\
\hline
\end{tabular}

according to IPA, two major pathways emerges: (I) cell-to-cell signaling and interaction, cell-mediated immune response, inflammatory response and (II) cell response to therapeutics.

known interactions reported in the literature. Results for the network analysis are displayed in Tables 1-3. Results for pathway analysis displayed in Figures 6 and 7.

\section{Discussion}

Quercitrin, which mostly exists in its glycoside form quercitrin?, is the most widely distributed flavonoid in nature. It possesses various potential properties including an antioxidant and anti-inflammatory for cell damage of $\beta$-cells; however, the underlying mechanisms leading to its anticancer effects are still unknown.

There are limited studies focusing on the anticancer effects of quercitrin in the literature. Epidemiologic studies have indicated that dietary flavonoids could present their effects in oral cancer. Browning et al. examined whether quercitrin could directly affect cell proliferation using human oral squamous carcinoma SCC-9 cells. They found that quercitrin showed no effect with minimal cellular uptake and no hydrolysis (19). Xu et al. studied the molecular mechanisms of apoptosis in prostate cancer induced by quercitrin and found that quercitrin exerted little effect and was not effective at inducing apoptosis (20). Ding et al. examined the effects of quercitrin on tumor promotion in mouse JB6 cells and quercitrin blocked TPA-induced neoplastic transformation in JB6 P+ cells (14). They also showed that quercitrin stimulated the activation of NFE2-related factor (Nrf2) and GST ARE luciferase activity and that it was able to block DNA damage induced by UVB (14).

In this study we determined that quercitrin has antiproliferative, cytotoxic, and apoptotic effects in NSCLC cell lines. Zheng et al. showed that quercetin revealed its anticancer effect by induction of apoptotic mechanism on A549 lung cancer cell lines in time- and dose-dependent manner in accordance with our results (21). In order to determine networks and pathways regulated by quercitrin treatment in NSCLC and lung fibroblast cells, we performed Illumina Human HT-12v4 beadchip microarrays on A549, NCI-H358, and MRC5 cells. After normalizing microarray data with Genome Studio, we used IPA software (Ingenuity Systems Inc.) to perform pathway analysis by comparing gene expression values between $50 \mu \mathrm{mol}$ quercitrin and untreated cells. We performed biological function analysis by comparing quercitrin treated and control fold change values and found that cancer, cell death, and cellular growth were the most statistically significant biofunctions among others in both A549 and NCI-H358 cell lines, respectively. After pathway analysis, we determined that leukocyte transendothelial migration, cell adhesion, phosphatidylinositol signaling system and DNA damage pathways were the most statistically significant pathways for both A549 and NCIH358 cell lines.

As a result, we found that quercitrin could help to induce cancer-related pathways and found that cellular development and apoptosis were closely related on NSCLC cells. The results indicated that quercitrin-induced antiproliferative effect could inhibite cell adhesion pathway, directing NSCLC cells to migration. We believe that these data will help to understand molecular mechanisms underlying quercitrin apoptotic effects.

Table 3. Literature-based clustering of differentially genes using an Ingenuity Pathway Analysis (IPA) after $48 \mathrm{~h}$ of $50 \mu$ mol quercitrin stimulation in cancer cells $^{\mathrm{a}}$

\begin{tabular}{|c|c|c|}
\hline Pathway name & Differentially expressed genes & Adjusted $p$ \\
\hline $\begin{array}{l}\text { Leukocyte transendothelial } \\
\text { migration }\end{array}$ & $\begin{array}{l}\text { ACTN1,ACTN4,CLDN10,CLDN23,CLDN4,CLDN8,F11 R,ITGB1,MSN,MYL2,MYL9,OCLN,PIK3R1, } \\
\text { PRKCB,PTK2B,RAP1A,ROCK1 }\end{array}$ & 0 \\
\hline Adherens junction & ACTN1,ACTN4,BAIAP2,CSNK2A1,CSNK2A2,IGF1R,INSR,LEF1,RAC3,SNAI2,SORBS1,SRC,TCF7,WAS & $4,30 \mathrm{E}-14$ \\
\hline $\begin{array}{l}\text { Antigen processing and } \\
\text { presentation }\end{array}$ & $\begin{array}{l}\text { CD74,CTSB,HLA-A,HLA-DQA1,HLA-DRB4,HLA- } \\
\text { E,HSP90AA1,HSPA1L,HSPA6,HSPA8,IFNA1,IFNA6,KIR2DL3,KIR2DL4,KIR2DS5,KIR3DL3,LLRC3,LTA }\end{array}$ & $6,54 \mathrm{E}-10$ \\
\hline $\begin{array}{l}\text { Phosphatidylinositol } \\
\text { signaling system }\end{array}$ & DGKQ,INPP4A,INPP5D,PIK3C2A,PIK3R1,PLCB2,PLCE1,PRKCB & $3,46 \mathrm{E}-03$ \\
\hline
\end{tabular}

${ }^{\mathrm{a}}$ According to IPA, two major pathways emerges: (I) Leukocyte transendothelial migration and (II) CAM adhesion molecules. 
ACBD6 acyl-CoA binding domain containing 6 ADPRH ADP-ribosylarginine hydrolase

ALKBH7 alkB, alkylation repair homolog ANAPC15 anaphase promoting complex subunit 15

C21 orf59 chromosome 21 open reading frame 59

C2orf49 chromosome 2 open reading frame 49

C6orf123 chromosome 6 open reading frame 123

C7orf10 chromosome 7 open reading frame 10

CEP95 centrosomal protein $95 \mathrm{kDa}$

COQ3 hexaprenyldihydroxybenzoate methyltransferase

CRYZL1 crystallin, zeta (quinone reductase)-like 1

CTDSPL2 CTD (carboxy-terminal domain, RNA polymerase II,

polypeptide A) small phosphatase like 2

DTWD1 DTW domain containing 1

ERI2 ERI1 exoribonuclease family member 2

FAHD2A fumarylacetoacetate hydrolase domain containing $2 \mathrm{~A}$

FAM216A family with sequence similarity 216 , member A

GOLIM4 golgi integral membrane protein 4

HNF4A hepatocyte nuclear factor 4 , alpha

KIAA0141 death ligand signal enhancer

KLHL28 kelch-like 28

LSMD1 LSM domain containing 1MRPL22

OGFOD2 2-oxoglutarate and iron-dependent oxygenase

domain containing 2

POC1A POC1 centriolar protein A

PRR3 pseudo-response regulator 3

RBAK RB-associated KRAB zinc finger

RBKS ribokinase

RIOK1 RIO kinase 1

SLC22A18 solute carrier family 22, member 18

SLC25A40 solute carrier family 25 , member 40

TMEM208 transmembrane protein 208

TMEM242 transmembrane protein 242

ZAN zonadhesin

ZNF502 zinc finger protein 502

ZSCAN18 zinc finger and SCAN domain containing 18

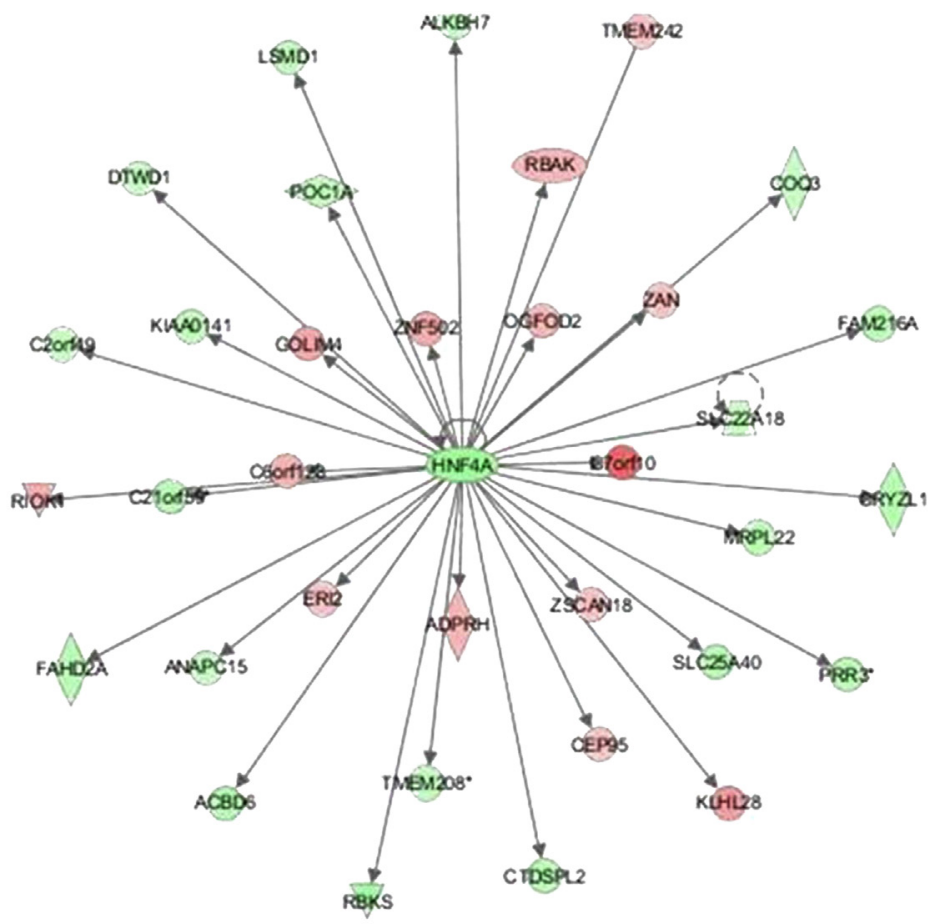

Figure 6. Functional analysis of the top selected genes identified. The network was algorithmically constructed by Ingenuity Pathway Analysis (IPA) software on the basis of the functional and biological connectivity of genes. HNF4A in central node generated by the Ingenuity Pathway Analysis (IPA) software are shown.

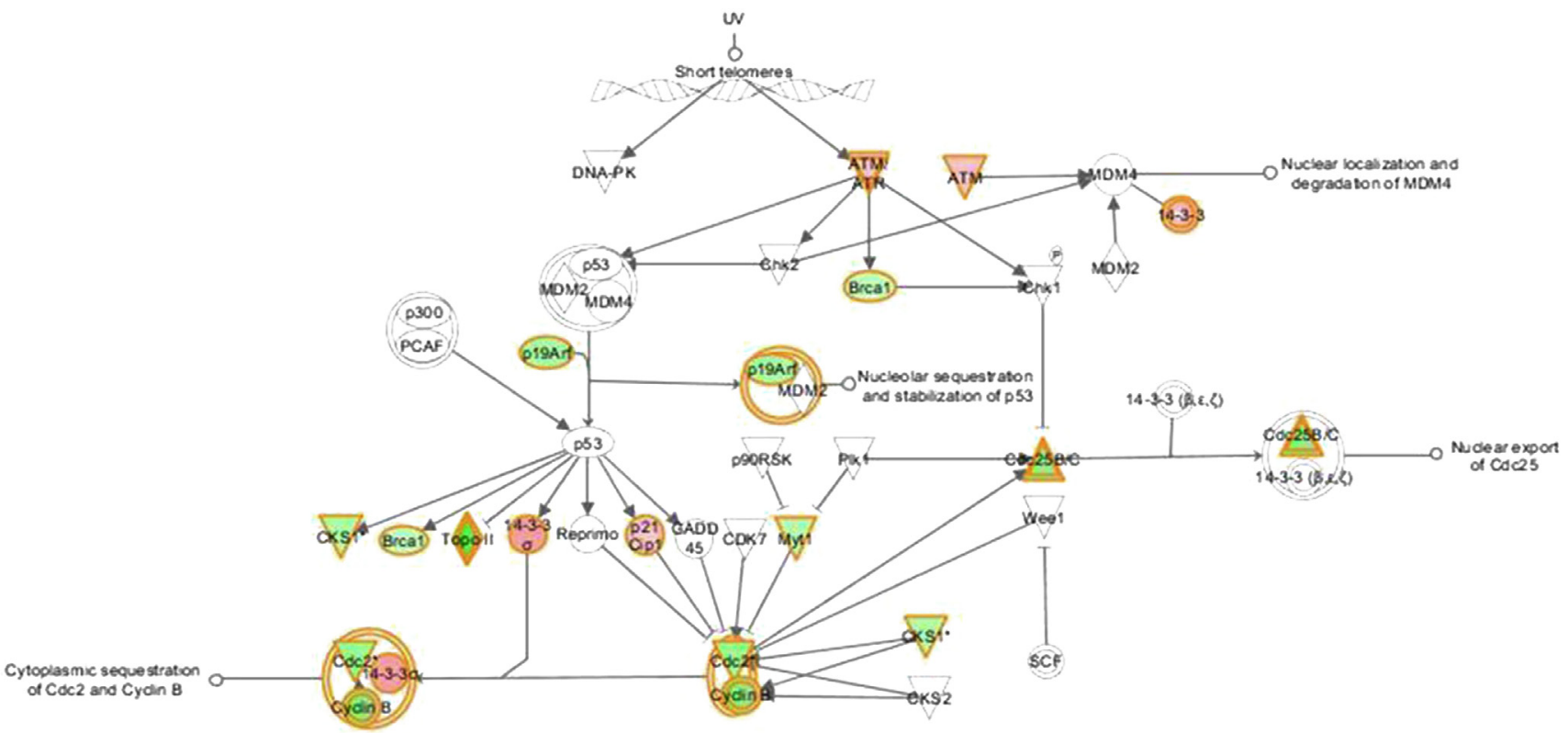

Figure 7. DNA Damage Signaling Network. Ingenuity pathway analysis (IPA) software was used to analyze identified genes involved in the DNA Damage pathway that were differentially expressed in A549, NCI-H358 cells. Genes labeled in red and green were those identified as up- and down-regulated and other genes were those related to the regulated genes on the basis of the network analysis. 


\section{Acknowledgments}

We would like to thank to Mr. David Chapman for English editing.

\section{Funding}

Istanbul University Scientific Research Projects, Number: 9205.

\section{Conflict of interest}

The authors declare that they have no financial disclosures or conflict of interest.

\section{References}

1. Pao W, Girard N. New driver mutations in non-small-cell lung cancer. Lancet Oncol 2011;12:175-180.

2. Travis WD. Classification of lung cancer. Semin Roentgenol 2011;46: $178-186$.

3. Brognard J, Clark AS, Ni Y, et al. Akt/protein kinase B is constitutively active in non-small cell lung cancer cells and promotes cellular survival and resistance to chemotherapy and radiation. Cancer Res 2001;61:3986-3997.

4. Schiller JH. Current standards of care in small-cell and non-small-cell lung cancer. Oncology 2001;1:3-13.

5. Gottschling S, Schnabel PA, Herth FJ, et al. Are we missing the target? Cancer stem cells and drug resistance in non-small cell lung cancer. Cancer Genomics Proteomics 2012;9:275-286.

6. Denlinger CE, Ikonomidis JS, Reed CE, et al. Epithelial to mesenchymal transition: the doorway to metastasis in human lung cancers. J Thorac Cardiovasc Surg 2010;140:505-513.

7. Goeckenjan G, Sitter H, Thomas M, et al, German Respiratory Society;German Cancer Society. Prevention, diagnosis, therapy, and follow-up of lung cancer: interdisciplinary guideline of the German Respiratory Society and the German Cancer Society. Pneumologie 2011;65:39-59;
Burris HA 3rd. Shortcomings of current therapies for non-small-cell lung cancer: unmet medical needs. Oncogene 2009;1:4-13.

8. Giaccone G. Twenty-five years of treating advanced NSCLC: what have we achieved? Ann Oncol 2004;4:81-83.

9. Neal JW, Sequist LV. Exciting new targets in lung cancer therapy: ALK, IGF-1R, HDAC, and Hh. Curr Treat Options Oncol 2011;11:36-44.

10. Sangha R, Lara PN Jr, Mack PC, et al. Beyond antiepidermal growth factor receptors and antiangiogenesis strategies for nonsmall cell lung cancer: exploring a new frontier. Curr Opin Oncol 2009;21:116-123.

11. Mateen S, Raina K, Agarwal R. Chemopreventive and anti-cancer efficacy of silibinin against growth and progression of lung cancer. Nutr Cancer 2013;1:3-11.

12. Nones JE, Spohr TC, Gomes FC. Quercitrin, a flavone glycoside, as mediator of neuronal survival. Neurochem Res 2011;36:1776-1784.

13. Tanaka T, Takahashi R. Flavonoids and asthma. Nutrients 2013;5: $2128-2143$.

14. Ding M, Zhao J, Bowman L, et al. Inhibition of AP-1 and MAPK signaling and activation of Nrf2/ARE pathway by quercitrin. Int J Oncol 2010;36:59-67.

15. Gee JM, DuPont MS, Rhodes MJ, et al. Quercetin glucosides interact with the intestinal glucose transport pathway. Free Radic Biol Med 1998;25:19-25.

16. Wagner C, Fachinetto R, Dalla Corte CL, et al. Quercitrin, a glycoside form of quercetin, prevents lipid peroxidation in vitro. Brain Res 2006; 1107:192-198

17. Cruz EA, Da-Silva SA, Muzitano MF, et al. Immunomodulatory pre treatment with Kalanchoe pinnata extract and its quercitrin flavonoid effectively protects mice against fatal anaphylactic shock. Int Immunopharmacol 2008;8:1616-1621.

18. Yang HM, Ham YM, Yoon WJ, et al. Quercitrin protects against ultraviolet B-induced cell death in vitro and in an in vivo zebrafish model. J Photochem Photobiol B 2012;114:126-131.

19. Browning AM, Walle UK, Walle T. Flavonoid glycosides inhibit oral cancer cell proliferation role of cellular uptake and hydrolysis to the aglycones. J Pharm Pharmacol 2005;57:1037-1042.

20. $\mathrm{Xu} \mathrm{R}$, Zhang $\mathrm{Y}$, Ye $\mathrm{X}$, et al. Inhibition effects andinduction of apoptosis of flavonoids on the prostate cancer cell line PC-3 in vitro. Food Chem 2013;138:48-53.

21. Zheng SY, Li Y, Jiang D, et al. Anticancer effect and apoptosis induction by quercetin in the human lung cancer cell line A-549. Mol Med Rep 2012;5:822-826. 